Rev. High Pressure Sci. Technol., Vol. 7 (1998) 1259 1261

\title{
Solvent Density Dependence of the Photolysis Quantum Yield in Supercritical Fluids
}

\author{
Y. Kimura, K. Sugihara, H. Ooe, M. Terazima, and N. Hirota \\ Department of Chemistry, Graduate School of Science, Kyoto University, Kyoto 606-01, Japan
}

\begin{abstract}
The transient grating (TG) method has been applied to studies on the photolysis quantum yield $\left(\phi_{d}\right)$ of iodine and diphenyl disulfide in supercritical fluids. The solvent density dependence of $\phi_{\mathrm{d}}$ for iodine obtained by the TG method is quite different from the result by the transient absorption method (B. Otto, et al., J. Phys. Chem., 81, 202 (1984)), if the TG signal is analyzed only by the thermal grating contribution. If we assume that the difference comes from the overestimate of $\phi_{d}$ from the TG method, the origin could be ascribed to the neglect of volume grating term. Under this assumption, the estimated reaction volume for the dissociation of iodine should be a considerably large negative over the density regions studied. [photolysis quantum yield, supercritical fluids, transient grating method, cage model, reaction volume]
\end{abstract}

\section{Introduction}

The photo-dissociation and recombination reaction is one of the simplest reactions which reflects various interesting aspects of the solvent effect: e.g., the collisional deactivation and recombination of the molecules on the dissociative potential surface before the complete dissociation (primary geminate recombination), the diffusive effect on the recombination of the produced geminate pair (secondary geminate recombination) and of the non-geminate pair, and so on. The photolysis quantum yield $\phi_{\mathrm{d}}$, defined as the fraction of the pair of the free radicals, is a characteristic quantity which reflects the solvent effect mentioned above. It is quite an interesting issue how $\phi_{d}$ depends on the solvent over a wide density region from the gaseous to liquid phases. Until now, however, studies on the density effect have been limited to diatomic cases such as iodine[1-4]. In this work, we present the results on the study of $\phi_{d}$ by the transient grating (TG) method, which detects the thermal energy dissipated by the recombination[5]. We have applied this method to the study of $\mathrm{I}_{2}$ and diphenyl disulfide (DPDS) in several supercritical fluids. DPDS is well known to undergo the photolysis of the S-S bond by the UV excitation forming phenylsulfanyl radicals, which recombine with the quantum yield almost unity[ 6-8].

\section{Theoretical basis}

In the TG measurement, the sample solution is irradiated by two laser beams crossing in the sample solution at the same time. This creates a light interference pattern in the sample solution. The TG intensity is monitored by the probe beam diffraction due to this grating. The basic formalism to analyze the TG signal for the photodissociation and recombination reaction is given by $\mathrm{Zhu}$ and Harris[5]. We have simplified their analysis, and already proved that our analytical procedure is well applicable to the system in liquid solution[9,10]. Briefly, the intensity of the TG signal $\left(\mathrm{I}_{\mathrm{TG}}\right)$ is determined by the following two terms as

$I_{T G} \propto(\Delta n)^{2}+(\Delta k)^{2}$,

where $\Delta \mathrm{n}$ is the peak-null difference of the refractive index, $\Delta \mathrm{k}$ is the peak-null difference of the absorption coefficient. The refractive index term $(\Delta n)$ is a sum of contributions from the thermal $\left(\Delta n_{\text {th }}\right)$, population $\left(\Delta n_{\text {pop }}\right)$, and volume $\left(\Delta n_{V}\right)$ gratings, and $\Delta \mathrm{k}$ consists of the population grating only as follows:

$\Delta n=\Delta n_{t h}+\Delta n_{p o p}+\Delta n_{V}$
$\Delta k=\Delta k_{p o p}$

The thermal grating signal originates from the heat expansion of the solvent by the photochemical reactions, and is expressed by

$$
\Delta n_{t h}(t)=\left(\frac{\mathrm{d} n}{\mathrm{~d} T}\right)\left[Q_{f} \exp \left(-D_{t h} q^{2} t\right)+\frac{\mathrm{d} Q_{S}(t)}{\mathrm{d} t} * \exp \left(-D_{t h} q^{2} t\right)\right]
$$

where $D_{t h}$ is the thermal diffusion constant, $q$ the grating lattice vector, and the product * the convolution integral, respectively. The first term represents the fast heat released after the photoexcitation such as the excess energy relaxation of the unbound state, the primary and secondary geminate recombination. The second term corresponds to the slow heat released by the nongeminate recombination. These are expressed explicitly as,

$Q_{f}=[\mathrm{C}]_{0}\left[E_{e x}-\Delta H \phi_{d}\right] /\left(\phi_{d} C_{P} \rho\right)$

$Q_{S}(t)=Q_{s} \frac{k_{\max } t}{1+k_{\max } t}$,

where $[C]_{0}$ is the number of parent molecule which dissociates to the radicals, $Q_{S} \equiv \Delta H[C]_{0} / \rho C_{P}$, and $k_{\max }=4 k_{\text {rec }}[C]_{0} . \quad E_{e x}$ is the excitation energy, $\Delta H$ the reaction enthalpy for the recombination reaction, $k_{r e c}$ the nongeminate recombination rate, $C_{P}$ the isobaric heat capacity, and $\rho$ the solvent density, respectively.

The population grating is due to the change of the molecular refractive index and is given by

$\Delta n_{p o p}(t)=\left(\Delta n_{p o p}^{P} / 2+\Delta n_{p o p}^{R}\right) N_{R}(t)$

where $\Delta n_{\text {pop }}{ }^{\mathrm{P}}$ and $\Delta \mathrm{n}_{\text {pop }}{ }^{\mathrm{R}}$ denote the change of the molecular refractive index term due to the decrease of the parent molecule and the increase of the radical molecule, respectively. $N_{R}(t)$ corresponds to the population decay of the radical given by

$N_{R}(t)=\frac{2[C]_{0}}{1+k_{\max } t}$

The volume grating is due to the reaction volume for the dissociation $(\Delta V)$, given by

$\Delta n_{V}(t)=\left(V \frac{\partial n}{\partial V} \Delta V / 2\right) N_{R}(t)$,

The reaction volume can be written using the equilibrium constant for the recombination, $\mathrm{K}$, as

$$
\begin{aligned}
\Delta V & =\kappa_{T} R T+\Delta V_{\text {conf }} \\
& =\kappa_{T} R T+\kappa_{T} R T \rho_{\mathrm{r}}\left(\frac{\partial \ln K}{\partial \rho_{\mathrm{r}}}\right),
\end{aligned}
$$


where $\kappa_{\mathrm{T}}$ is the isothermal compressibility of the solvent, and $\rho_{\mathrm{r}}$ is the reduced density by the critical density of the solvent. The first term is due to the increase of the translational mode by the dissociation, and the second term $\left(\Delta \mathrm{V}_{\text {conf }}\right)$, called as the configurational volume change, is due to the rearrangement of the solvent molecules by the dissociation.

The term due to the absorption coefficient in Eq. (3) consists of only the absorption term due to the population grating, and is given by an equation like Eq. (7).

Using the parameters by the above expressions, we can determine the photolysis quantum yield by

$$
\phi_{d}=\frac{E_{e x}}{\Delta H} \frac{Q_{S}}{Q_{S}+Q_{f}}
$$

We have used the value of $55 \mathrm{kcal} \mathrm{mol}^{-1}$ for DPDS and $36 \mathrm{kcal}$ $\mathrm{mol}^{-1}$ for $\mathrm{I}_{2}$ as $\Delta \mathrm{H}$, and neglected its density and solvent dependence.

\section{Experimental}

The experimental setup is described elsewhere[11]. Briefly, after two pump beams from an excimer laser (Lumonics Hyper $400 ; 308 \mathrm{~nm}$ ) was crossed in a sample solution at the same time, the diffraction of the probe beam (a He-Ne laser; $633 \mathrm{~nm}$ ) was monitored by a photomultiplier. A standard high pressure optical cell with two quartz windows was used for the measurement. In the study on the photolysis of iodine, the second harmonic of a Nd-YAG laser (Spectra Physic: GCR-170) was used for the source of the excitation, and the high pressure cell made of titanium was used.

\section{Results and Discussion}

Figure 1 shows the TG signals from $\mathrm{I}_{2}$ after the photoexcitation at $532 \mathrm{~nm}$ at several reduced densities of the solvent in $\mathrm{CO}_{2}$ at $323.2 \mathrm{~K}$. The TG signal has two components, the fast rise with instrumental response which corresponds to the fast decay processes such as the excess energy relaxation, the primary and secondary geminate recombination, and the slow rise component which derives from the non-geminate recombination.

Similar TG signals are observed for DPDS to those of $\mathrm{I}_{2}$ Figure 2 shows typical TG signals after the photo-excitation of DPDS in $\mathrm{C}_{2} \mathrm{H}_{6}$ at $323.2 \mathrm{~K}$. The signal rises slower in this case mainly due to the slower recombination reaction of phenylsulfanyl radical than iodine.

In the liquid solution, the TG signal was well analyzed by the

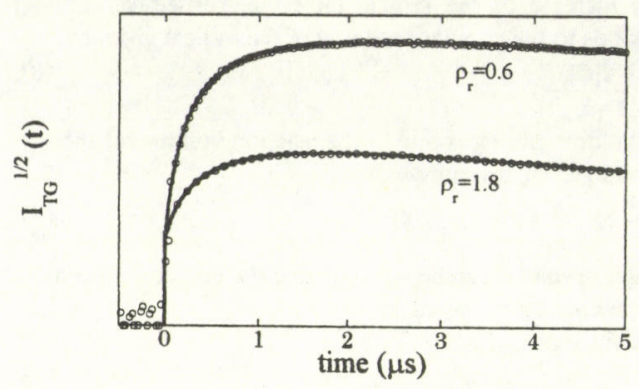

Fig. 1. Typical TG signals after the photoexcitation of $\mathrm{I}_{2}$ in $\mathrm{CO}_{2}$ at $323.2 \mathrm{~K}$ (circles). The solid lines denotes the simulated signals.

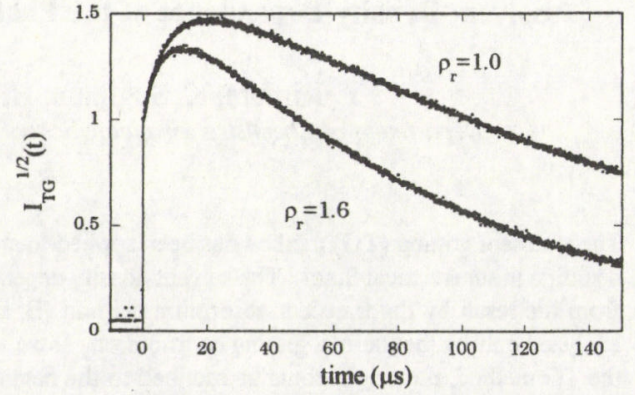

Fig. 2. Typical TG signals after the photoexcitation of DPDS in $\mathrm{C}_{2} \mathrm{H}_{6}$ at $323.2 \mathrm{~K}$ (dots). The solid lines denote the simluated signals.

term due to the thermal grating only $[5,9,10]$. We have obtained $\phi_{d}$ of $I_{2}$ as $0.30 \pm 0.03$ in hexane and $0.24 \pm 0.03$ in heptane by analyzing the TG signal only with the thermal grating term. These values are close to the values reported by $\mathrm{Zhu}$ and Harris using the same TG method $(0.25 \pm 0.03$ in hexane and $0.19 \pm 0.02$ in heptane)[5], and by Dutoit et al. using the transient absorption (TA) technique ( 0.23 in heptane) [4], but different from those reported by Luther et al. using the TA technique $(0.15 \pm 0.01$ in heptane)[12], and by Meadow and Noyes using the radical trapping method $(0.46 \pm 0.07)[13]$. We have also measured the photolysis quantum yield by the photoacoustic method[14], and obtained $\phi_{d}$ of $0.30 \pm 0.05$ in hexane[10].

Firstly, we have simulated the TG signals in fluids by the thermal grating term only, and the results are given in Fig. 3 against $T / \eta$, where $\eta$ is the viscosity of the solvent. In the figure, we have also plotted the results of $I_{2}$ determined by the TA method in ref. 1 . The viscosity dependence of $\phi_{d}$ for $I_{2}$ and DPDS obtained by the TG method is quite similar to each other. They do not show any significant viscosity effect in the lower viscosity region, and much enhanced effect in the higher viscosity region. However, our results for $\mathrm{I}_{2}$ are much larger than the values in ref. 1 especially in the medium-density region. The discrepancy indicates that either the TA method underestimated the quantum yield or the TG method overestimates the quantum yield. In the followings, we examine the latter possibility.

The most possible cause which makes the large difference in the medium-density region is the contribution of the volume grating neglected in our fitting procedure. In the medium-density region, the reaction volume may have a large value due to the large compressibility of the solvent (see Eq. (10)). Simple order estimation by Eq. (5) and Eq. (9) gives the volume grating as large as $25 \%$ of the thermal grating at the critical density of the solvent at $323 \mathrm{~K}$ in the case of the excitation light as $532 \mathrm{~nm}$. Therefore we have refit the TG signals for $I_{2}$ including the volume grating term. The separation between the thermal and the volume grating terms by the signal simulation was not always straightforward, and we found several local minima for the fitting parameters in some cases. To remove the ambiguity of the fitting, we have included the volume grating term so as to give the value of $\phi_{\mathrm{d}}$ determined by the TA measurement. In this way, the reaction volume for $\mathrm{I}_{2}$ at each solvent density is determined uniquely by the signal fitting. 


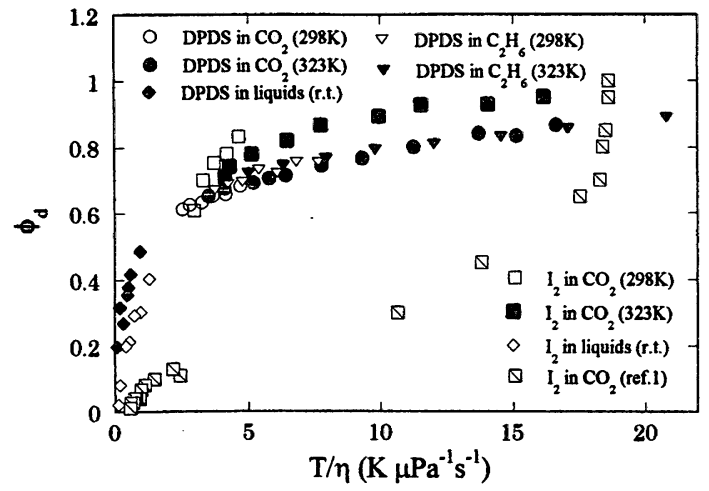

Fig. 3. Solvent viscosity dependence of $\phi_{d}$ for $I_{2}$ and DPDS in fluids at $323.2 \mathrm{~K}$, together with the results in liquid solvents.

Figure 4 shows the solvent density dependence of the reaction volume for the dissociation of $\mathrm{I}_{2}$ in $\mathrm{CO}_{2}$ at $323.2 \mathrm{~K}$. The reaction volume is calculated by the following relation between the total thermal signal and the volume grating signal at $t=0$, given by

$\Delta n_{V}(0) \gamma\left[\left(Q_{f}+Q_{s}\right)\left(\frac{\partial n}{\partial T}\right)\right]=\frac{\phi_{\mathrm{d}} \rho \mathrm{C}_{\mathrm{p}}}{\mathrm{E}_{\mathrm{ex}} \alpha_{\mathrm{T}}} \Delta V$

where $\alpha_{T}$ is thermal expansion coefficient. The estimated reaction volume in Fig. 4 is negative over the density regions studied, and the absolute value is larger at the medium-density region. The negative volume change for the dissociation is not so strange in the medium-density region. In the case of nitroso-compound, a large magnitude of a negative value of $\Delta \mathrm{V}_{\text {conf }}$ for the dissociation is observed in fluids at the medium-density region[15]. In that case, the negative reaction volume, or the positive volume change for the dimerization is explained by the desolvation effect due to the overlapping of the solvation shells during the course of the dimerization. In the case of $I_{2}$, however, $\Delta V$ itself is negative, and the absolute value seems extraordinary large. This suggests that a very large desolvation effect should exist for the dimerization process of $I_{2}$, since $\kappa_{T} R T$ always makes a positive contribution to the reaction volume for the dissociation. At the low density limit, the translational contribution will be dominant in the reaction volume, and $\Delta \mathrm{V}$ will be positive. However, the density dependence in Fig. 4 does not show any sign of tendency to be positive in the lower-density region studied here, which seems to contradict to the theoretical prediction. To check the validity of the estimated reaction volume, the study on the probe wavelength dependence, the study in the density region connecting the highest density of the present work with the liquid like density region, and the study in the very low density region are now under way.

In the case of DPDS, the effect of the reaction volume on the TG signal may be smaller than the case of $\mathrm{I}_{2}$, since the excitation energy is much (about two times) larger than the case of $I_{2}$. It is also to be noted that $\phi_{\mathrm{d}}$ for DPDS is well correlated with the solvent viscosity in a similar way both in $\mathrm{CO}_{2}$ and $\mathrm{C}_{2} \mathrm{H}_{6}$ (see Fig. 3 ), not with the solvent reduced density. If the volume grating term is dominant in the TG signal of DPDS, $\phi_{\mathrm{d}}$ may not be scaled by the viscosity, since the viscosity of $\mathrm{C}_{2} \mathrm{H}_{6}$ is about half of that of $\mathrm{CO}_{2}$ at the same reduced density. The viscosity dependence of $\phi_{\mathrm{d}}$

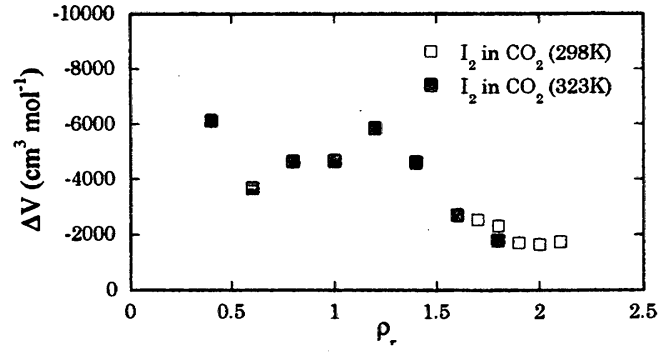

Fig. 4. The estimated reaction volume for the dissociation of $\mathbf{I}_{2}$.

like in Fig. 3 is predicted by the theory based on a cage model[1]. However, a possible existence of the contribution of the reaction volume may reduce the value of $\phi_{d}$ in a similar way to the case of $\mathrm{I}_{2}$ to some extent. To make a quantitative discussion, further information of the reaction volume will be required.

\section{Conclusion}

We have studied the photolysis quantum yield by the TG method. The quantum yield determined by analyzing the TG signal only with the thermal grating term seriously disagrees with that determined by the TA method. If the disagreement comes from the overestimation of the TG method, the origin should be attributed to the reaction volume in the TG signal. With incorporating this effect, we can get the density dependence of the reaction volume for the dissociation process of $I_{2}$. The estimated reaction volume for the dissociation is negative, which suggests a very strong desolvation effect.

\section{References}

[1] B. Otto, J. Schroeder, and J. Troe, J. Phys. Chem., 81, 202 (1984)

[2] J. Troe, J. Phys. Chem., 90, 357 (1986).

[3] H. Hippler, V. Schubert, and J. Troe, J. Chem. Phys., 81, 3931 (1984).

[4] J.-C. Dutoit, J.-M. Zellweger, and H. van den Bergh, J. Chem. Phys., 78, 1825 (1983).

[5] X. R. Zhu and J. M. Harris., Chem. Phys., 157, 409 (1991).

[6] F. C. Thyrion, J. Phys. Chem., 77, 1478 (1973).

[7] T. W. Scoot and S. N. Liu, J. Phys. Chem., 93, 1393 (1989).

[8] Y. Hirata, Y. Niga., M. Ohta., M. Takizawa, and T. Okada, Res. Chem. Intermed., 21, 823 (1995).

[9] Y. Kimura, K. Sugihara, M. Terazima, and N. Hirota, Bull. Chem. Soc. Jpn. 70, 2657 (1997).

[10] H. Ooe, Y. Kimura, M. Terazima, and N. Hirota, in preparation.

[11] D. Kanda, Y. Kimura, M. Terazima and N. Hirota, Ber. Bunsenges. Phys. Chem., 100, 656 (1996).

[12] K. Luther, J. Schroeder, J. Troe, and U. Unterberg, J. Phys. Chem., 84, 3072 (1980).

[13] L. F. Meadow and R. M. Noyes, J. Am. Chem. Soc., 82, 1872 (1959)

[14] M. Terazima and T. Azumi, Bull. Chem. Soc. Jpn. 62, 2862 (1989).

[15] Y. Kimura and Y. Yoshimura, J. Chem. Phys., 96, 3085 (1992). 